\section{DNA MODIFICATION}

\section{Pass it along}

Cell https://doi.org/10.1016/j.cell.2018.11.002 (2018)

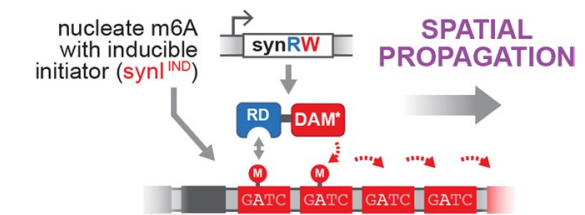

CLUSTERED REPORTER

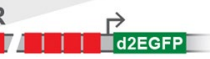

Credit: Elsevier

Chemical modifications on DNA and histones play important roles in a wide range of biological processes, and can also enable gene expression memory (epigenetics). It is challenging to study the underlying mechanism of epigenetic regulation because of the complexity of natural chromatin networks. To overcome this problem, Park et al. utilized a synthetic biology approach to construct de novo an epigenetic regulatory system based on $N^{6}$-methyladenine $\left(\mathrm{m}^{6} \mathrm{~A}\right)$ in mammalian cells. They attached a variant of an Escherichia coli DNA adenine methyltransferase (Dam) to an engineered zinc finger protein or dCas9 to generate a synthetic initiator module (synI). SynI can bind to specific sequences and introduce $\mathrm{m}^{6} \mathrm{~A}$ at nearby GATC motifs in the promoter region of a reporter gene. When combined with engineered $\mathrm{m}^{6} \mathrm{~A}$ reader modules, their systems can activate or repress the expression of the reporter. They also constructed a three-module circuit by introducing an additional read-write (RW) module, which can propagate $\mathrm{m}^{6} \mathrm{~A}$ modifications along DNA and also maintain epigenetic memory during cell division. This synthetic approach provides a unique capability to study the quantitative design principles underlying chromatin spreading and heritable gene expression memory. YS

https://doi.org/10.1038/s41589-018-0221-2

\section{MICROBIAL METABOLISM}

\section{Cutting in choline}

Nat. Microbiol. 4, 155-163 (2019)

Elevated levels of circulating trimethylamine $\mathrm{N}$-oxide (TMAO) are associated with multiple human diseases. TMAO arises from the oxidation of trimethylamine (TMA), which can be produced from choline by certain gut microorganisms harboring the choline utilization (cut) operon. To understand how these microbes obtain choline, Chittim et al. first tested representative gut bacteria for their ability to process cholinecontaining substrates, finding several strains that could cleave the membrane lipid phosphatidylcholine (PC) to choline. Bioinformatic analysis led to the identification of a phospholipase $\mathrm{D}$ (PLD)-encoding gene present only in PC-metabolizing strains. Deletion of this gene in an Escherichia coli isolate abolished the strain's ability to produce choline or TMA from PC and to use PC as a carbon source. In vitro biochemical characterization of the E. coli PLD validated PC as the enzyme's preferred substrate, while further bioinformatic analyses and mutagenesis implicated an active site glutamate residue as important for substrate selectivity. As these PLDs are widespread
UBIQUITINATION

Ras tuning
Science 362, 1171-1177 (2018) Science 362, 1177-1182 (2018)

LZTR1 is an adaptor for the CUL3 ubiquitin ligase complex, and mutations in LZTR1 have been identified in liver and brain cancers and Noonan syndrome; however, the exact molecular role of LZTR1 in these disorders was not clear. Bigenzahn et al. used haploid genetic screens to connect LZTR1 with regulation of MAPK signaling and resistance to tyrosine kinase inhibitors. To identify substrates of LZTR1-containing complexes, Stekov et al. utilized a mass spectrometry approach that traps protein complexes in viral particles, whereas Bigenzahn et al. used a proximity biotinylation proteomic approach to detect complexes of LZTR1 and the main RAS GTPase family members $\mathrm{K}-, \mathrm{N}-$ and HRAS. Formation of this complex resulted in the ubiquitination of RAS proteins, decreasing RAS activity and MAPK signaling. LZTR1 pathogenic mutations reduced binding to CUL3 and RAS, interfering with RAS ubiquitination and leading to enhanced RAS protein levels and plasma-membrane localization. Interestingly, Stekov et al. found that HRAS gets ubiquitinated at K170, and molecular simulations with lipidated RAS supported the notion that $\mathrm{K} 170$ ubiquitination prevented membrane association. Overall, these findings reveal a new mechanism of RAS regulation providing a potential explanation for LZTR1 mutations in human disease.

https://doi.org/10.1038/s41589-018-0219-9

among choline-utilizing gut bacteria, distinct from those PLDs found in pathogens, and insensitive to human PLD inhibitors, their identification may help in the development of new selective therapeutics.

https://doi.org/10.1038/s41589-018-0218-X

\section{IMMUNITY \\ A new Tar-get for inhibition Nature 563, 705-709 (2018)}

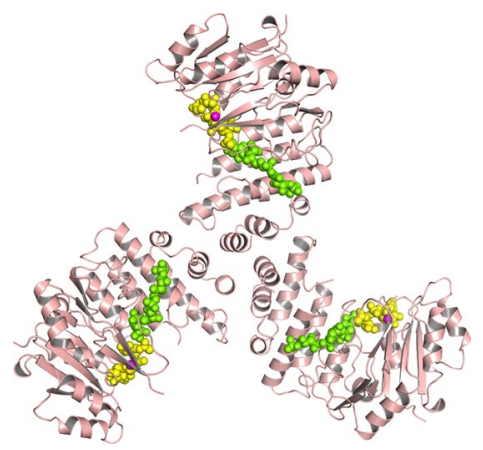

Credit: Nature

Methicillin-resistant Staphylococcus aureus (MRSA) strains are thought to succeed by evading the host immune system. Though humans have antibodies against $S$. aureus, a successful vaccine has not been developed. Most of these host antibodies are directed against wall teichoic acid (WTA), a ribitolphosphate (RboP) surface polymer modified by $N$-acetylglucosamine (GlcNAc). To better understand the basis of increased fitness and pathogenicity, Gerlach et al. examined S. aureus genomes for paralogs of WTA biosynthesis genes and found a protein, TarP, encoded by three $S$. aureus prophages with similarity to the standard WTA glycosyltransferase TarS. TarP is sufficient for WTA glycosylation and for conferring several WTA functional properties, although it catalyzes glycosylation of RboP at a different site compared to TarS. A structural study of TarP with WTA could explain this alternate WTA glycosylation and a reduced affinity of TarS for WTA compared to TarP. Finally, the authors found that, compared to TarP, TarS more strongly increases IgG binding and immunogenicity. These results suggest that TarP-expressing S. aureus are less susceptible to detection and elimination by the host immune system and reveal a new target for vaccines and for small molecule inhibition.

$M B$

https://doi.org/10.1038/s41589-018-0220-3

Mirella Bucci, Caitlin Deane, Grant Miura and Yiyun Song 